\title{
Low-Complexity Digital Predistortion For Reducing Power Amplifier Spurious Emissions in Spectrally-Agile Flexible Radio
}

\author{
Mahmoud Abdelaziz ${ }^{1}$, Lauri Anttila ${ }^{1}$, Joseph R.Cavallaro ${ }^{2}$, Shuvra S. Bhattacharyya ${ }^{3}$, Abbas Mohammadi ${ }^{4}$, \\ Fadhel Ghannouchi ${ }^{5}$, Markku Juntti ${ }^{6}$ and Mikko Valkama ${ }^{1}$ \\ ${ }^{1}$ Department of Electronics and Communications Engineering, Tampere University of Technology, Tampere, Finland \\ ${ }^{2}$ Rice University, ECE Department, Houston, TX \\ ${ }^{3}$ University of Maryland, ECE Department, College Park, MD \\ ${ }^{4}$ Department of Electrical Engineering, Amirkabir University of Technology, Tehran, Iran \\ ${ }^{5}$ iRadio Lab, Department of Electrical and Computer Engineering, University of Calgary, Alberta, Canada \\ ${ }^{6}$ University of Oulu, Dept. Communications Engineering, Oulu, Finland \\ E-mail: mahmoud.abdelaziz@tut.fi
}

\begin{abstract}
Increasing the flexibility in spectrum access is a key to enhanced efficiency in radio spectrum utilization. Noncontiguous carrier aggregation (CA) is one enabling technology towards more flexible spectrum access, but can also lead to serious implementation challenges in terms of transmitter linearity. Especially when a single power amplifier (PA) is deployed for all carriers, serious intermodulation components will rise which can easily limit the maximum transmit power. In this paper, a low-complexity digital predistortion (DPD) solution is developed to reduce the most critical spurious components at the PA output, opposed to more classical full bandwidth linearization. The developed concept and associated parameter learning and optimization are particularly tailored towards mobile devices, building on limited narrowband feedback receiver with reduced instrumentation complexity and reduced-rate DPD processing. The developed DPD solution can handle PAs with substantial memory effects, and is verified with extensive simulation examples in various non-contiguous carrier aggregation scenarios and practical PA models with memory.
\end{abstract}

Index Terms-Carrier aggregation, cognitive radio, digital predistortion, flexible spectrum access, intermodulation, LTEAdvanced, mobile transmitter, power amplifier

\section{INTRODUCTION}

Cognitive radio (CR) systems seek to increase the efficiency of radio spectrum utilization by allowing secondary user (SU) access to spatially and/or temporally unused spectral chunks in limited geographical areas such that interference towards primary users (PUs) is kept within the specified limits [1], [2]. At physical layer, this calls for frequency-agile flexible radio transceivers which can transmit and receive spectrally wellcontained waveforms, including also non-contiguous trans-

This work was supported in part by the US National Science Foundation under grants CNS-1265332 and CNS-1264486 as well as by the Finnish Funding Agency for Technology and Innovation (Tekes) through the TRIAL programme under the project "Cross-Layer Modeling and Design of EnergyAware Cognitive Radio Networks (CREAM)". The work was also funded by the Academy of Finland under the project \#251138 "Digitally-Enhanced RF for Cognitive Radio Devices" and the Linz Center of Mechatronics (LCM) in the framework of the Austrian COMET-K2 programme. mission with multiple simultaneous carriers. Similar noncontiguous or scattered spectrum access scenarios are also emerging in 3GPP mobile cellular radio, most notably in LTEAdvanced in terms of non-contiguous intra-band and interband carrier aggregation (CA) [3].

In above-kind flexible spectrum access scenarios, the linearity of transceiver circuits, and especially transmit power amplifier (PA), is one key challenge [4]. Especially with single PA based transmitter architecture, non-contiguous transmission can result in severe intermodulation components stemming from the nonlinear characteristics of the PA. These spurious components, if not properly controlled, can easily violate the SU-PU interference constraints in cognitive radio systems [5] or the spurious emission limits of commercial mobile cellular radio such as 3GPP LTE-Advanced [6], [7]. One alternative to reduce the spurious emissions is simply to backoff the transmit power, called Maximum Power Reduction (MPR) in 3GPP LTE uplink context. As shown through actual RF measurements in [7] with an LTE-Advanced Release 12 intraband CA signal composed of two fully allocated $5 \mathrm{MHz}$ carriers and $30 \mathrm{MHz}$ separation, the needed MPRs can be even in the order 10-15 dB, which has then a very big impact on the achievable transmission rate and coverage.

Instead of backing-off the power, a fascinating alternative is to deploy digital predistortion (DPD) for suppressing the unwanted spurious emissions [8]-[12]. In CA transmitters, however, the total bandwidth of the digital signal is already very high and running classical full-bandwidth DPD processing may not be feasible, especially in mobile devices [9]. Thus, alternative reduced-complexity DPD solutions are needed. In the existing literature, some works towards sub-band specific linearization have been reported in the recent years. In [10] and [11], DPD solutions with separate processing for the fundamental bands and the higher-order intermodulation (IM) bands in CA transmitter context were developed. Quasimemoryless model for each subband was assumed and the 
parameter estimation was based on off-line measurements with a large-signal network analyzer (LSNA). Memory polynomial type predistortion of dual-band PAs, focusing on the spectral regrowth mitigation of the component carriers only, was in turn proposed in [13], and then extended to cover also the third-order IM bands in [12] but including only memoryless processing. All these works allow lowering the sample rate in the DPD processing, compared to classical full-bandwidth solutions. In [12] and [13], also the feedback receiver instrumentation is simplified as only the linearized sub-bands need to be measured. The reported solutions are, however, limited either to simple memoryless processing or assume offline measurements and parameter optimization.

In this article, opposed to offline measurements based parameter estimation, we develop an online reduced complexity predistortion solution, specifically tailored to suppress thirdorder spurious IM components in non-contiguous CA transmitters. First, detailed modeling for the spurious components is carried out assuming a general case of PA with memory effects. Then, stemming from this modeling, a specific cancellation signal is injected into the transmit path such that the thirdorder spurious IM component at PA output is suppressed. The properties of this injected cancellation signal are adaptively controlled, deploying a decorrelation-based adaptive learning algorithm and narrowband observation receiver with reduced instrumentation complexity. Compared to the previous works in [10], [11], and [12], explicit knowledge of the PA properties is then not needed. Comprehensive simulation experiments are also reported, with practical wideband PA models, evidencing efficient suppression of the selected spurious components. The developed solution thus offers an efficient low-complexity DPD solution for reducing PA-induced spurious emissions in spectrally-agile flexible radio systems.

\section{Spurious Component Modeling AND Digital PREDISTORTION}

\section{A. Spurious IM3 Component Modeling for Power Amplifiers with Memory}

In [14], modeling of the spurious IM3 component of a third order memoryless PA was performed. This analysis established the basis for the memoryless decorrelating DPD using the relevant third order basis functions, and it is shortly reiterated next. The modeling is carried out at composite baseband equivalent level, and the two component carriers (CC) are assumed to be separated by $2 f_{I F}$. Defining the composite baseband equivalent input and output signals of the $\mathrm{PA}$ respectively as

$$
\begin{aligned}
& x(n)=x_{1}(n) e^{j 2 \pi \frac{f_{I F}}{f_{s}} n}+x_{2}(n) e^{-j 2 \pi \frac{f_{I F}}{f_{s}} n} \\
& y(n)=\beta_{1} x(n)+\beta_{3}|x(n)|^{2} x(n),
\end{aligned}
$$

the PA output signals at the spurious IM3 bands centered at $+/-3 f_{I F}$ read

$$
\begin{aligned}
& y_{I M 3_{+}}(n)=\beta_{3}\left(x_{2}^{*}(n) x_{1}^{2}(n)\right) \\
& y_{I M 3_{-}}(n)=\beta_{3}\left(x_{1}^{*}(n) x_{2}^{2}(n)\right) .
\end{aligned}
$$

Here, $\beta_{1}$ and $\beta_{3}$ are the (unknown) memoryless PA coefficients, and $x_{1}(n)$ and $x_{2}(n)$ are the baseband equivalents of the input CCs.

Now, in order to extend this analysis to power amplifiers exhibiting memory effects, we shall assume a third order Parallel Hammerstein (PH) PA model excited with the same dual-carrier signal. The PA output signal in this case is

$$
y(n)=f_{1}(n) \star x(n)+f_{3}(n) \star|x(n)|^{2} x(n),
$$

where $f_{1}(n)$ and $f_{3}(n)$ are the filters in the main and third order $\mathrm{PH}$ branches, respectively, and $\star$ is the convolution operator. Through direct substitution of (1) in (4), the baseband equivalent positive and negative IM3 terms, located in the composite BB equivalent at three times the IF frequency, can be easily extracted and read

$$
\begin{aligned}
& y_{I M 3_{+}}(n)=f_{3}^{+}(n) \star\left(x_{2}^{*}(n) x_{1}^{2}(n)\right) \\
& y_{I M 3_{-}}(n)=f_{3}^{-}(n) \star\left(x_{1}^{*}(n) x_{2}^{2}(n)\right) .
\end{aligned}
$$

Here, $f_{3}^{+}(n)$ is the baseband equivalent response of $f_{3}(n)$ at the positive $I M 3$ band $\left(+3 f_{I F}\right)$, and similarly, $f_{3}^{-}(n)$ is the baseband equivalent response of $f_{3}(n)$ at the negative $I M 3$ band $\left(-3 f_{I F}\right)$. These are formally defined as

$$
\begin{aligned}
& f_{3}^{+}(n)=L P F\left\{e^{-j 2 \pi \frac{3 f_{I F}}{f_{s}} n} f_{3}(n)\right\} \\
& f_{3}^{-}(n)=L P F\left\{e^{j 2 \pi \frac{3 f_{I F}}{f_{s}} n} f_{3}(n)\right\},
\end{aligned}
$$

with $L P F\{$.$\} denoting an ideal low pass filtering operation,$ with a passband 3 times the bandwidth of the wider $\mathrm{CC}$. While the PA output signal contains also other signal and distortion terms, our objective is to develop a low-complexity DPD solution that aims at reducing only these particular IM3 components, and thus assist the mobile transceiver to fulfill the spurious emission mask with a smaller MPR. This is formulated next at structural level in Section II-B while the actual parameter optimization and practical estimation through the decorrelation principle are addressed in Section III.

\section{B. Proposed DPD Structure for Dedicated Spur Reduction}

To simplify the presentation, the following analysis will focus on canceling only the $I M 3_{+}$term in (5). In short, the idea is to inject a proper additional low-power cancellation signal to (1), located at $+3 f_{I F}$, such that the level of the $I M 3_{+}$term at PA output is reduced. Stemming from the signal structure in (5), a natural injection signal is a filtered version of $x_{2}^{*}(n) x_{1}^{2}(n)$ using a filter $\alpha(n)$ composed of a set of $\mathrm{N}+1$ taps $\alpha_{0}, \alpha_{1}, \ldots \alpha_{N}$. Incorporating such DPD processing, the composite baseband equivalent PA input signal now reads

$$
\begin{aligned}
\tilde{x}(n)= & x_{1}(n) e^{j 2 \pi \frac{f_{I F}}{f_{s}} n}+x_{2}(n) e^{-j 2 \pi \frac{f_{I F}}{f_{s}} n} \\
& +\left[\alpha(n) \star\left(x_{2}^{*}(n) x_{1}^{2}(n)\right)\right] e^{j 2 \pi \frac{3 f_{I F}}{f_{s}} n} .
\end{aligned}
$$

Here, and in the continuation, we use $(\tilde{.})$ variables to indicate DPD processing and corresponding predistorted signals. 
Substituting now $\tilde{x}(n)$ in (4), the most dominant terms at the positive IM3 band at the PA output read

$$
\begin{aligned}
& \tilde{y}_{I M 3_{+}}(n)=\left(f_{3}^{+}(n)+f_{1}^{+}(n) \star \alpha(n)\right) \star x_{2}^{*}(n) x_{1}^{2}(n) \\
& +2 f_{3}^{+}(n) \star\left[\left(\left|x_{1}(n)\right|^{2}+\left|x_{2}(n)\right|^{2}\right)\left(\alpha(n) \star x_{2}^{*}(n) x_{1}^{2}(n)\right)\right] .
\end{aligned}
$$

From (9), it can be shown that the strength of the considered IM3 band at the PA output depends directly on, and can thus be controlled by, the DPD coefficients $\alpha_{0}, \alpha_{1}, \ldots \alpha_{N}$. In the next section, we address practical feedback-based adaptive learning of $\alpha(n)$ for efficient online IM3 mitigation.

\section{AdAPtiVE DECORRELATION-BASED LEARNING OF DPD PARAMETERS}

Below we address the practical estimation of the DPD parameters. As in the previous section, we focus mostly on the positive IM3 spurious band to keep the notations compact. Similar processing and estimation can be directly developed then also for the corresponding negative IM3 spurious band.

\section{A. 3rd Order Inverse Solution}

Examining (9), it can be seen that the solution that nulls the third order term is simply obtained by setting the first line of the equation to zero, i.e., $f_{3}^{+}(n)+f_{1}^{+}(n) \star \alpha_{i n v}(n)=$ $0, \forall n$. Unlike in the memoryless scenario, in order to solve this equation for the DPD filter $\alpha(n)$, it is easier to transform the expression to the frequency domain where convolutions are turned into multiplications. Consequently, the 3rd order inverse solution for $\alpha(n)$ in the frequency domain becomes

$$
A_{i n v}\left(e^{j \frac{2 \pi f}{f_{s}}}\right)=-\frac{F_{3}^{+}\left(e^{j \frac{2 \pi f}{f_{s}}}\right)}{F_{1}^{+}\left(e^{j \frac{2 \pi f}{f_{s}}}\right)},
$$

where $F_{1}^{+}\left(e^{j \frac{2 \pi f}{f_{s}}}\right)$ and $F_{3}^{+}\left(e^{j \frac{2 \pi f}{f_{s}}}\right)$ are the baseband equivalent frequency responses of the $\mathrm{PH}$ filters $f_{1}(n)$ and $f_{3}(n)$ at the positive IM3 band (i.e. $+3 f_{I F}$ ). After that, an IDFT operation can be performed on $A_{i n v}\left(e^{j \frac{2 \pi f}{f_{s}}}\right)$ in order to obtain the impulse response of the DPD filter $\alpha_{i n v}(n)$.

Clearly, the 3rd order inverse solution implies estimating $F_{1}^{+}\left(e^{j \frac{2 \pi f}{f_{s}}}\right)$ and $F_{3}^{+}\left(e^{j \frac{2 \pi f}{f_{s}}}\right)$, which is not a simple task in practice. Such estimation for a memoryless DPD was proposed in [10], [12]. In this article, instead of explicitly trying to estimate the 3rd order inverse solution, we propose multi-tap adaptive decorrelation-based DPD as an effective solution. The actual estimation procedure is addressed next.

\section{B. Decorrelation-Based Adaptive Parameter Estimation}

Examining the first line of (9), the dominant term in the positive IM3 expression is $\left(f_{3}^{+}(n)+f_{1}^{+}(n) \star \alpha(n)\right) \star x_{2}^{*}(n) x_{1}^{2}(n)$. As shown in the previous subsection a suitable linear filter $\alpha_{i n v}(n)$ can remove the dominant third-order term. However, applying predistortion always creates higher-order IM products on the IM3 bands, such as the fifth order term seen on the second line of (9), that the 3rd order inverse solution cannot suppress. This fifth order term can be seen to be strongly correlated with the dominant third order basis function $x_{2}^{*}(n) x_{1}^{2}(n)$.
We therefore conjecture, that decorrelating the IM3 band signal at the PA output with $x_{2}^{*}(n) x_{1}^{2}(n)$ and its delayed replicas could give superior performance compared to the 3 rd order inverse solution. This approach was originally developed in [14] for a memoryless DPD and PA. There, the adaptive decorrelation algorithm was shown to clearly improve the IM3 band reduction compared to the 3rd order inverse solution, and was also shown to work under 5th order PA nonlinearity. The memoryless solution from [14] is here generalized to a multitap solution, aiming for improved spur reduction with practical wideband PAs exhibiting substantial memory effects.

As implicated by the analyses in Sections II-B and III-A, the proposed multi-tap adaptive decorrelation DPD is formulated as a multi-tap adaptive filter which filters the locally generated Static Non-Linear (SNL) basis function $x_{2}^{*}(n) x_{1}^{2}(n)$, with the objective of decorrelating it with the IM3 band signal at the PA output. Consequently, the adaptive decorrelation coefficient update reads as follows:

$$
\begin{aligned}
e(n) & =\tilde{y}_{I M 3_{+}}(n) \\
u(n) & =x_{2}^{*}(n) x_{1}^{2}(n) \\
\underline{u}(n) & =\left[\begin{array}{llll}
u(n) & u(n-1) & \ldots u(n-N)
\end{array}\right]^{T} \\
\underline{\alpha}(n) & =\left[\begin{array}{lll}
\alpha_{0}(n) & \alpha_{1}(n) \ldots \alpha_{N}(n)
\end{array}\right]^{T} \\
\underline{\alpha}^{*}(n+1) & =\underline{\alpha}^{*}(n)-\frac{\mu}{\|\underline{u}(n)\|^{2}+C} \underline{u}(n) e^{*}(n) .
\end{aligned}
$$

Where $\mathrm{N}+1$ denotes the filter length. The scaling factor $\|\underline{u}(n)\|^{2}+C$ of the adaptation constant $\mu$ in (15) corresponds to the Normalized Least Mean Square (NLMS) scaling factor and the constant $\mathrm{C}$ is added to avoid numerical problems when the energy in the current set of samples is very small.

The proposed adaptive learning structure is shown in Fig. 1. Notice that the learning and the actual main DPD path filtering are separated, to be able to handle the delay of the predistortion feedback loop. This delay is denoted by the block $z^{-L}$ in front of the 'Adaptive Decorrelator' block.

\section{Computational and Hardware Complexity}

Compared to conventional full-band DPD, the proposed technique has many benefits. First, the computational requirements of the DPD processing are greatly reduced. Full-band DPD with nonlinearity order 3 would need to run at a sample rate 3 times the composite dual-carrier signal bandwidth, which quickly becomes impossible when increasing carrier separation. The minimum sample rate for the proposed technique is only 3 times the bandwidth of the wider component carrier. To give an example, assume a CA scenario with two $10 \mathrm{MHz}$ carriers separated by $100 \mathrm{MHz}$. The minimum sample rate with 3rd order full-band DPD would be $330 \mathrm{MHz}$, whereas with the proposed technique, it is only $30 \mathrm{MHz}$.

Second, the proposed technique implies very simple instrumentation complexity for the feedback receiver, which is needed for parameter learning with unknown PA characteristics, as only narrowband feedback capturing the considered IM3 spurious band is needed. Using the same numerical example as above, and the feedback structure shown in Fig. 


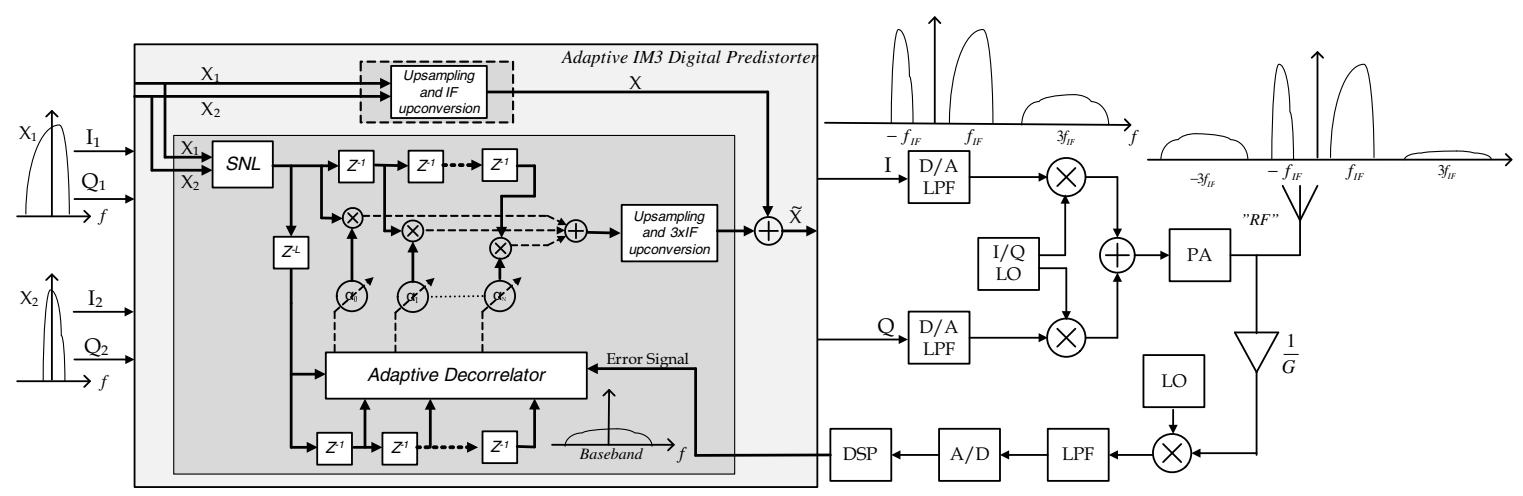

Fig. 1. Principal adaptive DPD system architecture for third-order spurious intermodulation reduction in a dual-carrier transmitter. Also essential composite baseband equivalent spectra are shown.

1, conventional full-band DPD would need an ADC with a minimum sample rate of $660 \mathrm{MHz}$, while the proposed technique needs only a $60 \mathrm{MHz}$ ADC.

Third, the complexity of the proposed structure could be further reduced, if the signal injection, which in Fig. 1 is fully digital, would be implemented in the analog domain using an extra DAC and I/Q modulator for each injected signal. This would also enable using separate DAC's for the component carriers instead of a single wideband DAC. Sample rate requirements at the digital side and DAC stage would thus be reduced, which would otherwise inevitably become a bottleneck if the carrier separation was increased beyond couple of tens of MHz. This approach, however, implies complex cost-related trade-offs between digital and analog instrumentation, and requires careful study to find the carrier separation after which analog injection becomes cheaper. Such a discussion is out of the scope of this article and remains an important topic for future work.

To shortly demonstrate the differences in hardware complexity between the fully digital implementation shown in Fig. 1 and the analog injection technique, assume again a CA scenario with two $10 \mathrm{MHz}$ carriers separated by $100 \mathrm{MHz}$. The fully digital injection would need a dual DAC running at a minimum of $330 \mathrm{MHz}$, and on the digital side, three complex upsamplers to bring the sample rate to $330 \mathrm{MHz}$, plus two adders running at this frequency. Using analog injection, one would need a dual DAC at $110 \mathrm{MHz}$ for the combined main carriers (or alternatively two $10 \mathrm{MHz}$ dual DAC's for the main carriers separately), one dual DAC at $30 \mathrm{MHz}$ for the injection signal, plus one to three analog I/Q modulators and one or two adders, depending on the main carriers' DAC implementation.

\section{Simulation Results And Analysis}

\section{A. Simulation Scenarios}

So far, the theoretical foundation of the decorrelating DPD algorithm has been established along with an implementation proposal for linearizing power amplifiers exhibiting memory effects as shown in Fig. 1. In this section, a non-contiguous dual-carrier signal is used to test the proposed DPD concept.
As a concrete example, the two carriers are separated by 60 $\mathrm{MHz}$ and both are deploying QPSK carrier modulation with flexible carrier bandwidth. This models an example flexible spectrum access scenario, applicable already in 3GPP LTEAdvanced Release 12.

The DPD analysis and derivations in section II were formulated for a parallel Hammerstein power amplifier model. However, for a more general and realistic testing scenario in which there is a mismatch between the PA model in the analysis and actual verification simulations, we will also deploy a Wiener power amplifier model, in addition to parallel Hammerstein, in our simulations. Furthermore, the channel bandwidth assignment of the two carriers is varied in order to test the performance of the proposed DPD algorithm in flexible channel assignment scenarios where the bandwidth allocation of the two CCs can be symmetrical or asymmetrical.

For simplicity, we focus in the forthcoming examples mostly on the positive IM3 band, but like mentioned already in the previous sections, the negative IM3 band can also be considered and processed similarly by just changing the SNL basis in Fig. 1 from $x_{2}^{*}(n) x_{1}^{2}(n)$ to $x_{1}^{*}(n) x_{2}^{2}(n)$ and then upconverting the DPD injection signal to the negative IM3 band instead of the positive one. Finally, in the feedback path, appropriate downconversion and filtering to observe the negative IM3 signal from the PA output, instead of the positive one, is then obviously also needed. An example of this is given in subsection $\mathrm{C}$ below.

\section{B. Simulation Results with 3rd Order PA}

First, a third-order Wiener amplifier is tested with an IIP3 of $+17 \mathrm{dBm}$ and transmission power of $+22 \mathrm{dBm}$ thus implying only $1 \mathrm{~dB}$ back off (MPR) from the maximum 3GPP LTE class $3 \mathrm{UE}$ transmission power $(+23 \mathrm{dBm})$. Asymmetric spectrum access is considered, with $5 \mathrm{MHz}$ and $10 \mathrm{MHz}$ carrier bandwidths. Fig. 2 shows the PSD of the PA output signal under three different conditions. First, without using any DPD, then using the single-tap decorrelating DPD, and finally using the proposed multi-tap decorrelating DPD with five taps. The learning in the adaptive DPD solutions is iterated for approx- 
imately 300 microseconds before freezing the coefficients. As the figure demonstrates, there is a clear gain in the suppression of the positive IM3 band when the multi-tap algorithm and processing is deployed, compared to the single tap one. This is mainly because the single-tap DPD cannot process frequencydependent IMD within the IM3 band, imposed by PA memory.

Another simulation scenario is next considered and demonstrated where the PA model is a parallel Hammerstein one. In this case, for demonstration purposes, very long memory is imposed in the PA model corresponding to high frequency selectivity within the IM3 band. In this scenario, both access carriers have an identical bandwidth of $5 \mathrm{MHz}$. Again the DPDs are adapted for approximately 300 microseconds, and the corresponding output spectra are shown in Fig. 3. Again the proposed multi-tap processing yields substantially improved reduction of the considered spurious component, compared to memoryless processing.

\section{Simulation Results with 5th Order PA}

Real-world practical PAs usually exhibit higher-order nonlinearities than just the third order. Using similar notation as in the previous sections, the wideband output signal of a 5-th order parallel Hammerstein PA model is of the form

$$
\begin{aligned}
y(n) & =f_{1}(n) \star x(n)+f_{3}(n) \star|x(n)|^{2} x(n) \\
& +f_{5}(n) \star|x(n)|^{4} x(n),
\end{aligned}
$$

where in addition to $f_{1}(n)$ and $f_{3}(n), f_{5}(n)$ is now the filter in the fifth order PH branch. One can then easily show that without any DPD processing, the positive IM3 band baseband equivalent reads

$$
\begin{aligned}
y_{I M 3_{+}}(n) & =f_{3}^{+}(n) \star\left(x_{2}^{*}(n) x_{1}^{2}(n)\right) \\
& +3 f_{5}^{+}(n) \star\left(\left|x_{2}(n)\right|^{2}\left(x_{2}^{*}(n) x_{1}^{2}(n)\right)\right) \\
& +2 f_{5}^{+}(n) \star\left(\left|x_{1}(n)\right|^{2}\left(x_{2}^{*}(n) x_{1}^{2}(n)\right)\right) .
\end{aligned}
$$

Here, $f_{5}^{+}(n)$ is the baseband equivalent response of $f_{5}(n)$ at the positive $I M 3$ band $\left(+3 f_{I F}\right)$. Thus, the IM3 band clearly now contains other signal terms in addition to the third order term $x_{2}^{*}(n) x_{1}^{2}(n)$ as a result of the fifth order distortion in the PA. However, we can also see that the additional fifth order terms in the IM3 band have a significant correlation with delayed replicas of the SNL basis function $x_{2}^{*}(n) x_{1}^{2}(n)$, thus justifying the application of the decorrelating solution also with higher-order PA models. A more extensive study for analyzing the output of a fifth order PA when the DPD injection is inserted is left for future work and here below we simply focus on experimental verification and demonstration.

Stemming from above, in order to shortly elaborate the applicability of the proposed multi-tap decorrelating DPD, a fifth-order Wiener PA model is now deployed in the simulator. The used PA model has $+17 \mathrm{dBm}$ IIP3 and $+26.5 \mathrm{dBm} 1 \mathrm{~dB}$ compression point, and includes clear memory effects. As in the third-order PA example, both the single and multi-tap DPD solutions are tested. The resulting convergence curves of the five-tap filter coefficients are shown in Fig. 4. Again, after approximately 300 microseconds, the coefficients are converged and can thus be frozen. The corresponding PA output PSD results are shown in Fig. 5, where it is again evident that the proposed multi-tap DPD solution outperforms the single-tap counterpart also in this practical scenario with higher-order PA model. In this example, both positive and negative IM3 spurious components are suppressed. We also show in the figure the general ITU spurious emission mask [15], namely $-30 \mathrm{dBm}$ over $1 \mathrm{MHz}$ measurement bandwidth. As the figure illustrates, the proposed five-tap DPD solution clearly allows the device to meet the spurious emission limits which in turn are violated not only without DPD but also with memoryless DPD processing. Notice also that as the DPD processing is optimized for third-order IMD reduction, the fifth-order spurs at IM5 bands are somewhat increased but still well within the spurious emission limits.

\section{CONCLUSIONS AND DiscusSiON}

A low complexity digital predistortion technique for wideband power amplifiers with memory was proposed, specifically designed for spectrum-agile mobile devices deploying noncontiguous carrier aggregation. The predistortion processing mainly targets to suppress the third-order intermodulation components which can fall into the critical spurious emission region when the component carriers are, e.g., a few tens of $\mathrm{MHz}$ apart. Novel parameter learning and optimization based on a narrowband observation receiver and decorrelating principle was devised, covering frequency-dependent multi-tap processing against PA memory within the spur bandwidth. The overall DPD concept was succesfully tested and demonstrated with different power amplifier models exhibiting memory effects. In addition, flexible bandwidth assignments for the transmitted component carriers were tested and demonstrated, which is an essential element in emerging flexible spectrum access and cognitive radio scenarios. In cognitive radio context, successful spur reduction, through DPD processing, allows for improved coexistence with other higher priority primary users while still meeting the interference limits imposed on the mobile transmitter output at adjacent channels and other protected parts of the spectrum. Without sufficient spectral containment of the secondary user signals, due to e.g. spurious emissions of the PA, SU-PU interference constraints may considerably compromise the deployment possibilities of dynamic spectrum access and in particular non-contiguous spectrum access. Thus, embedding an adaptive low-complexity DPD into the devices to improve the spectral containment of the signals is one of the enabling elements of dynamic and flexible spectrum access in the future. Our future work will focus on more comprehensive testing and performance evaluations of the proposed DPD concept, including both real-time hardware demonstrations and practical RF measurements.

\section{REFERENCES}

[1] S. Haykin, "Cognitive radio: brain-empowered wireless communications," IEEE J. Sel. Areas Commun, vol. 23, no. 2, pp. 201-220, Feb. 2005.

[2] I. Akyildiz, W.-Y. Lee, M. Vuran, and S. Mohanty, "Next generation/dynamic spectrum access/cognitive radio wireless networks: A survey," Computer Networks, vol. 50 no.13, pp. 2127-2159, Sept. 2006. 


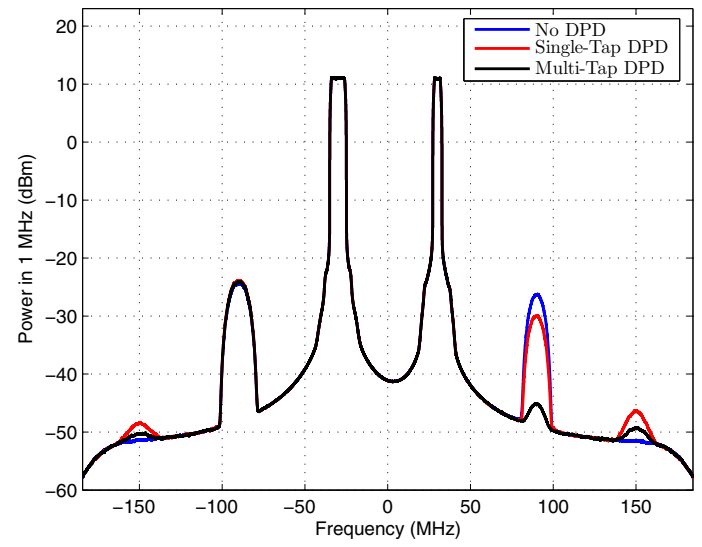

Fig. 2. Dual-carrier mobile transmitter baseband equivalent power spectra with asymmetrical channel $\mathrm{BW}$ assignments. 5 and $10 \mathrm{MHz}$ are allocated to the first and second CC respectively. The memoryless single tap and the proposed multi-tap adaptive DPD are compared. Third order Wiener PA is used, with $+17 \mathrm{dBm}$ IIP3 and output power of $+22 \mathrm{dBm}$.

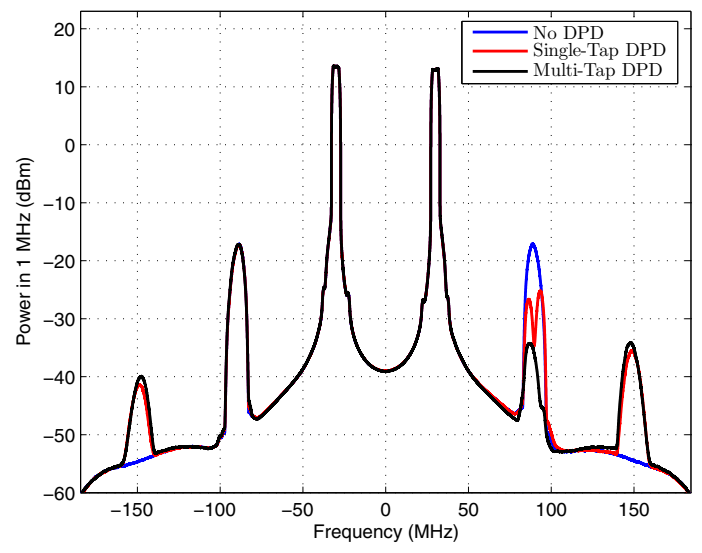

Fig. 3. Dual-carrier mobile transmitter baseband equivalent power spectra with symmetrical $5 \mathrm{MHz}$ allocation per $\mathrm{CC}$. The memoryless single tap and the proposed multi-tap adaptive DPD are compared. Third order PH PA is used with output power of $+22 \mathrm{dBm}$.

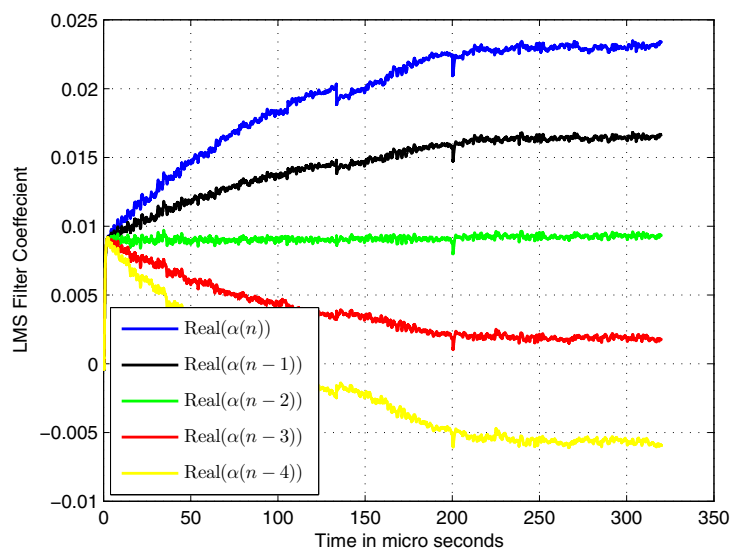

Fig. 4. Convergence of the multi-tap adaptive DPD coefficients in case of fifth-order Wiener PA.

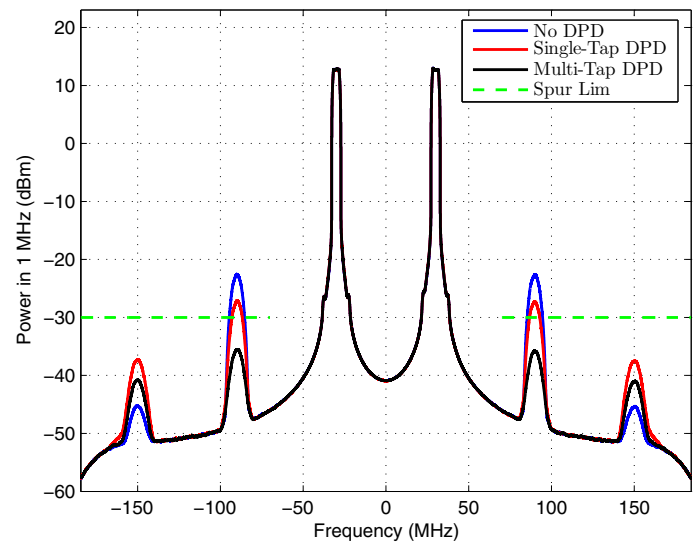

Fig. 5. Dual-carrier mobile transmitter baseband equivalent power spectra with symmetrical $5 \mathrm{MHz}$ allocation per CC. The memoryless single tap and the proposed multi-tap adaptive DPD are compared. Fifth order Wiener PA is used, with $+17 \mathrm{dBm}$ IIP3, $+26.5 \mathrm{dBm} 1 \mathrm{~dB}$ compression point and output power of $+22 \mathrm{dBm}$

[3] 3GPP Evolved Universal Terrestrial Radio Access (E-UTRA); User Equipment (UE) radio transmission and reception. http://www.3gpp.org/ftp/Specs/html-info/36101.htm, Std.

[4] B. Razavi, "Cognitive radio design challenges and techniques," IEEE J. Solid-State Circuits, vol. 45, no. 8, p. 15421553, 2010.

[5] R. Zhang, "On peak versus average interference power constraints for protecting primary users in cognitive radio networks," IEEE Trans. Wireless Commun., vol. 8, no. 4, pp. 2112-2120, April 2009.

[6] V. Lehtinen, T. Lähteensuo, P. Vasenkari, A. Piipponen, and M. Valkama, "Gating factor analysis of maximum power reduction in multicluster LTE-A uplink transmission," in Proc. IEEE Radio and Wireless Symposium (RWS), Austin, TX, Jan. 2013.

[7] T. Lähteensuo, "Linearity requirements in LTE Advanced mobile transmitter," Master's thesis, Tampere University of Technology, Tampere, Finland., May 2013. http://dspace.cc.tut.fi/dpub/handle/123456789/21504.

[8] J. Thorebäck, "Digital predistortion the evolution of linearized transmitters for radio basestations," in Signal Processing for Amplifiers Workshop, Chalmers, Sweden, Nov. 2012.

[9] M. Abdelaziz, A. Ghazi, L. Anttila, J. Boutellier, T. Lähteensuo, X. Lu, J. Cavallaro, S. Bhattacharyya, M. Juntti, and M. Valkama, "Mobile transmitter digital predistortion: Feasibility analysis, algorithms and design exploration," in 47th Asilomar Conf. Signals Systems Computers, Pacific, Grove, CA, USA, Nov. 2013.

[10] P. Roblin, S. K. Myoung, D. Chaillot, Y. G. Kim, A. Fathimulla, J. Strahler, and S. Bibyk, "Frequency-selective predistortion linearization of RF power amplifiers," IEEE Trans. Microw. Theory Tech., vol. 56, pp. 65-76, Jan. 2008

[11] J. Kim, P. Roblin, D. Chaillot, and Z. Xie, "A generalized architecture for the frequency-selective digital predistortion linearization technique," IEEE Trans. Microw. Theory Tech., vol. 61, pp. 596-605, Jan. 2013.

[12] S. Bassam, M. Helaoui, and F. Ghannouchi, "Channel-selective multicell digital predistorter for multi-carrier transmitters," IEEE Trans. Microw. Theory Tech., vol. 60, pp. 2344-2352, Aug. 2012.

[13] S. Bassam, F. Ghannouchi, and M. Helaoui, "2-D Digital Predistortion (2-D-DPD) architecture for concurrent dual-band transmitters," IEEE Trans. Microw. Theory Tech., vol. 59, pp. 2547-2553, Oct. 2011.

[14] M. Abdelaziz, L. Anttila, A. Mohammadi, F. Ghannouchi, and M. Valkama, "Reduced-complexity power amplifier linearization for carrier aggregation mobile transceivers," in Proc. IEEE International Conference on Acoustics, Speech and Signal Processing (ICASSP '14), Florence, Italy, 2014, (Accepted).

[15] International Telecommunication Union Radio Communication Sector, Recommendation ITU-R SM.329-12 Unwanted emissions in the spurious domain. http://www.itu.int/dms_pubrec/itur/rec/sm/R-REC-SM.32912-201209-I!!PDF-E.pdf, Std. 